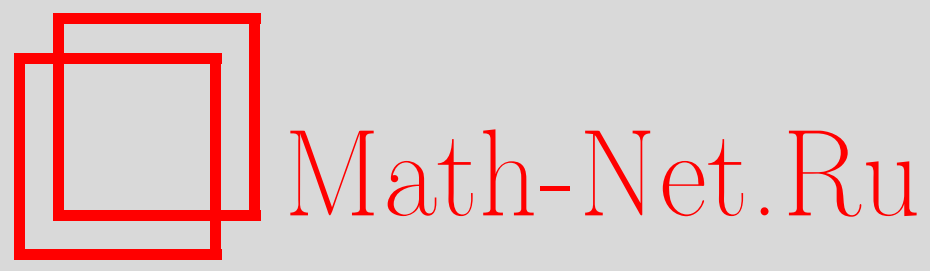

С. В. Кисляков, Н. Я. Кругляк, Устойчивость аппроксимации под действием сингулярных интегральных операторов, Функи. анализ и его прил., 2006, том 40, выпуск 4, 49-64

DOI: https://doi.org/10.4213/faa848

Использование Общероссийского математического портала MathNet.Ru подразумевает, что вы прочитали и согласны с пользовательским соглашением

http://www.mathnet.ru/rus/agreement

Параметры загрузки:

IP : 54.197 .130 .99

26 апреля 2023 г., 13:45:37

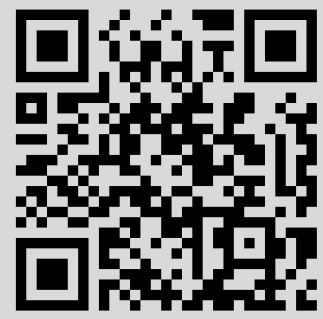


Функииональный анализ и его приложения

2006, т. 40, вып. 4, с. 49-64

УДК 517.9

\title{
Устойчивость аппроксимации под действием сингулярных интегральных операторов*
}

\author{
(c) 2006. С. В. Кисляков, Н. Я. Кругляк
}

Посвящается памяти Бориса Яковлевича Левина

Эта статья безнадежно вещественна, однако у нее есть комплексные истоки. О них нельзя не сказать, учитывая повод, по которому статья написана. Эти истоки лежат в теории интерполяции аналитических пространств Харди и им подобных.

Интерполяция (мы обсуждаем лишь вещественный метод) - это, по существу, наука о разбиении функций в сумму двух слагаемых, величина которых оценивается в разных нормах. Пусть этим нормам соответствуют (квази)банаховы пространства $X$ и $Y$, и предположим, что все рассматриваемые функции заданы на границе плоской области. Для простоты пусть даже эта область - круг $\mathbb{D}=\{z:|z|<1\}$. Тогда ее граница - это окружность $\mathbb{T}=$ $\{z:|z|=1\}$. Довольно часто удается корректно ввести «аналитические подпространства» $X_{A}$ и $Y_{A}$ в $X$ и $Y$, состоящие (мы намеренно избегаем точных деталей) из сужений на окружность функций, аналитических в круге. Например, $\left(L^{p}(\mathbb{T})\right)_{A}$ - это обычный класс Харди $H^{p}(\mathbb{T})$.

Предположим, что функция $f$ на окружности разбита в сумму, $f=g+h$, где $g \in X, h \in Y$. Всегда ли можно заставить слагаемые лежать в пространствах $X_{A}$ и $Y_{A}$, не слишком испортив их нормы, если заранее известно, что $f$ - сужение на границу некоторой функции, аналитической в круге? Иными словами, всегда ли при разложении в сумму можно сохранить аналитичность, не проиграв в оценках?

Дадим точную постановку, относящуюся к более общей ситуации. Пусть $\left(E_{0}, E_{1}\right)$ - интерполяционная пара, а $F_{0}$ и $F_{1}$ - замкнутые подпространства в $E_{0}$ и $E_{1}$. Пусть $f \in F_{0}+F_{1}$ и имеется разбиение $f=g+h$, где $g \in E_{0}$, $h \in E_{1}$. Всегда ли можно найти другое разбиение $f=\tilde{g}+\tilde{h}$, где $\tilde{g} \in F_{0}, \tilde{h} \in F_{1}$ и $\|\tilde{g}\|_{E_{0}} \leqslant C\|g\|_{E_{0}},\|\tilde{h}\|_{E_{1}} \leqslant C\|h\|_{E_{1}}(C$ не зависит от участвующих векторов)? Если так можно сделать всегда, то пара $\left(F_{0}, F_{1}\right)$ называется $K$-замкнутой в паре $\left(E_{0}, E_{1}\right)$.

Для пространств аналитических функций $K$-замкнутость изучалась подробно, начиная с работ Пизье [1], [2]. Как выяснилось, она - явление скорее обычное, чем исключительное (см. [3]-[6] и обзоры [7], [8]). Рассмотрим, например, весовые пространства $L_{w}^{p}(\mathbb{T})=\left\{f: f w \in L^{p}(\mathbb{T})\right\}, 0<p \leqslant \infty$, и соответствующие им аналитические подпространства $\left(L_{w}^{p}(\mathbb{T})\right)_{A}=H_{w}^{p}(\mathbb{T})$ (от веса требуется лишь суммируемость логарифма; заметим, что вес появляется сам собой при

*Работа поддержана Королевской Шведской академией наук. Исследования первого автора поддержаны РФФИ, грант 05-01-0924. 
конформной пересадке в круг невесовых классов $H^{p}$ из области со спрямляемой границей). В [3] показано, что пара $\left(H_{w_{0}}^{p_{0}}(\mathbb{T}), H_{w_{1}}^{p_{1}}(\mathbb{T})\right) K$-замкнута тогда и только тогда, когда $\log w_{0} w_{1}^{-1} \in \mathrm{BMO}$. Последнее условие, кстати, заведомо выполнено при $w_{0}=w_{1} \equiv 1$, т. е. для обычных пространств $H^{p}(\mathbb{T})$. Этим дело не ограничивается - если не выдумывать экзотических норм, пара $\left(X_{A}, Y_{A}\right)$ оказывается $K$-замкнутой для большинства стандартных пространств Кёте $X$ и $Y$. Методы, позволяющие все это доказать, разнообразны и относительно просты (см. указанные выше ссылки), однако по своей природе комплексны.

В статье [4] Бургейн предложил чисто вещественный метод доказательства $K$-замкнутости. Для пространств типа Харди этот метод не дает столь общих результатов - в сущности, он ориентирован лишь на классические пространства $H^{p}(\mathbb{T})$. Однако он имеет далеко идущие обобщения за пределы теории классов Харди. Остановимся на этом подробнее.

Обозначим через $\mathbb{P}$ проектор Рисса, т. е. ортогональный проектор пространства $L^{2}(\mathbb{T})$ на $H^{2}(\mathbb{T}), \mathbb{P} f=\sum_{n \geqslant 0} \hat{f}(n) z^{n}$, где через $\hat{f}(n)$ обозначен $n$-й коэффициент Фурье функции $f$. Общеизвестно, что этот оператор проектирует $L^{p}(\mathbb{T})$ на $H^{p}(\mathbb{T})$ при $1<p<\infty$, но разрывен при $p=1$ или $p=\infty$. Поэтому $K$-замкнутость пары $\left(H^{p_{0}}, H^{p_{1}}\right)$ в $\left(L^{p_{0}}, L^{p_{1}}\right)$ очевидна при $1<p_{0}<p_{1}<\infty$ (если $f \in H^{p_{0}}+H^{p_{1}}=H^{p_{0}}$ и $f=g+h$, где $g \in L^{p_{0}}, h \in L^{p_{1}}$, то нужное разложение в сумму аналитических слагаемых дается формулой $f=\mathbb{P} f=\mathbb{P} g+\mathbb{P} h)$. Трудности возникают, если $p_{0}=1$, или $p_{1}=\infty$, или, тем более, если выполнено и то, и другое. Выясняется, однако, что здесь достаточно рассматривать пары индексов вида $(1, p)$, где $p<\infty$ (бесконечный индекс затем включается в игру с помощью двойственности и других соображений общего характера; см. [5], [7]). Бургейн нашел способ доказать $K$-замкнутость пары $\left(H^{1}, H^{p}\right)$, использующий лишь то обстоятельство, что $\mathbb{P}$ - сингулярный интегральный оператор типа Кальдерона-Зигмунда.

Операторы Кальдерона-Зигмунда встречаются часто, и метод Бургейна применим во всех таких ситуациях. Приведем иллюстративный пример. Прежде, однако, изменим слегка точку зрения. Вопрос о $K$-замкнутости пары $\left(H^{1}, H^{p}\right)$ эквивалентен следующему. Пусть $f \in H^{1}+H^{p}=H^{1}(\mathbb{T})$ (т. е. $f \in L^{1}(\mathbb{T})$ и $\mathbb{P} f=f$ ), и пусть $t>0$. Верно ли, что расстояние (измеренное в $L^{1}$ ) от $f$ до шара радиуса $t$ в $L^{p}$ реализуется (с точностью до мультипликативной константы) на аналитической функции? Именно в такой форме мы будем ставить задачу в дальнейшем.

Теперь опишем обещанный пример. На пространстве $\mathbb{R}^{n}$ рассмотрим суммируемые градиентные векторные поля $F=\left(f_{1}, \ldots, f_{n}\right)(F=\nabla u$ для некоторого распределения $u$ и $\left.f_{j} \in L^{1}\left(\mathbb{R}^{n}\right)\right)$. Пусть $t>0$. Рассмотрим векторные поля $G$, почти реализующие $L^{1}$-расстояние от $F$ до шара радиуса $t$ в $L^{p}$ :

$$
G \in B_{L^{p}}(c t), \quad\|F-G\|_{L^{1}} \leqslant C \operatorname{dist}_{L^{1}}\left(F, B_{L^{p}}(t)\right) .
$$

(Здесь и далее через $B_{X}(\tau)$ обозначается шар радиуса $\tau$ с центром в нуле в банаховом пространстве $X$.) Найдется ли среди таких полей градиентное?

Ответ положителен и получается так. Рассмотрим пространство $\mathscr{H}$ всех квадратично-суммируемых векторных полей на $\mathbb{R}^{n}$, а в нем - подпространство $G$ градиентных векторных полей. Ортогональный проектор пространства $\mathscr{H}$ на $G$ нетрудно выписать явно в терминах преобразований Фурье. Из этого 
явного представления видно, что он - оператор типа Кальдерона-Зигмунда. Остается применить конструкцию Бургейна.

В этой статье мы будем заниматься следующей задачей. Пусть $X$ - пространство (векторных) функций на $\mathbb{R}^{n}$ (считаем, что их значения лежат в фиксированном евклидовом пространстве $H$ ). В качестве $X$ могут выступать пространства $L^{p}\left(\mathbb{R}^{n} ; H\right), p>1$ (как было только что), однако в большей степени нас интересуют классы гладких функций - например, однородные пространства Липшица и Соболева, пространство ВМО и т. п.

Предположим, что $T$ - сингулярный интегральный оператор на $H$-значных функциях (точные условия на $T$ будут приведены ниже). Мы отказываемся от требования, чтобы оператор $T$ был проектором. Пусть $f$ - такая $H$-значная функция, что $f, T f \in L^{1}\left(\mathbb{R}^{n} ; H\right)$. Мы хотим приблизить (в метрике пространства $L^{1}$ ) функцию $f$ функцией $g$ из шара $B_{X}(c t)$ почти оптимальным образом, но так, чтобы при этом и функция $T g$ была почти оптимальным $L^{1}$-приближением для $T f$. Схематически результаты будут выглядеть так.

Предположсим, что $f, T f \in L^{1}\left(\mathbb{R}^{n}, H\right)$. Тогда при каждом $t>0$ существует такая функиия $f_{t} \in B_{X}(c t)$, что

$$
\begin{gathered}
\left\|f-f_{t}\right\|_{L^{1}} \leqslant C \operatorname{dist}_{L^{1}}\left(f, B_{X}(t)\right) \\
\left\|T f-T f_{t}\right\|_{L^{1}} \leqslant C\left(\left\|f-f_{t}\right\|_{L^{1}}+\operatorname{dist}_{L^{1}}\left(T f, B_{X}(t)\right)\right) .
\end{gathered}
$$

Оператор $T$ во всех наших рассмотрениях окажется ограниченным в пространстве $X$, так что автоматически $T f_{t} \in B_{X}\left(c^{\prime} t\right)$. Если $T-$ проектор и $T f=f$, то второе неравенство в (1) принимает особенно простой вид — как в обсуждавшихся выше примерах.

Заслуживает немедленного упоминания следующая деталь: функиия $f_{t}$ не зависит от оператора $T$ и находится практически конструктивно - с помощъю специального алгоритма, обобщающего классическое разложение Кальдерона-Зигмунда. Алгоритм этот зависит от пространства $X$ и при $X=L^{p}$, в сущности, выступает в изначальной форме, впервые появившейся в [9]. Подходящие обобщения процедуры Кальдерона-Зигмунда для ситуации, когда $X$ - то или иное пространство гладких функций, были построены в [10], [11]. В приложении в конце статьи мы помещаем конструкцию для $X=\operatorname{Lip}_{\alpha}$, публиковавшуюся ранее лишь в препринте [12].

В этой работе мы ограничимся двумя случаями: $X=L^{p}\left(\mathbb{R}^{n}, H\right), 1<p<\infty$ $(\S 1)$, и $X=\operatorname{Lip}_{\alpha}\left(\mathbb{R}^{n}, H\right)(\S 2)$. Материал $\S 1$ можно рассматривать как серию замечаний о методе Бургейна - среди прочего, мы придадим этому методу обещанный выше «алгоритмический» вид. Мы извлечем также следствия, которые потребуются в §2. Они, на наш взгляд, интересны и сами по себе (см. следствие 2 об одновременной аппроксимации и лемму 1 о двойственности).

$\S 2$ - главная часть статьи. В техническом плане самое важное здесь - это новая оценка для сингулярных интегралов (см. п. 2.3.1).

Имеются два препринта [12], [13], к которым можно обратиться за дополнительной информацией (например, о соболевских классах или о классе ВМО в роли пространства $X)$. Там же можно прочитать некоторые доказательства, не вошедшие в эту статью из-за недостатка места. При этом содержание статьи препринтами не покрывается. Новы, например, замечания из §3 о тригонометрической системе и о базисах из вейвлетов. Не говорится в [12], [13] и о векторных функциях. Однако дополнительных осложнений в векторном случае 
почти не возникает; поэтому в дальнейшем мы будем приводить для простоты лишь скалярные формулировки. Чтобы включить векторный случай, достаточно всюду (в том числе и в [12], [13]) воспринимать символ $|f(x)|$ не как модуль, а как поточечно вычисленную норму в пространстве $H$.

\section{§1. Пространство $L^{p}(\mathbb{R})$}

1.1. Алгоритм Кальдерона-Зигмунда для пары $\left(L^{1}, L^{\infty}\right)$. Под «кубом» мы понимаем куб со сторонами, параллельными координатным осям. В классической статье [9] для каждой функции $f \in L^{1}\left(\mathbb{R}^{n}\right)$ и каждого $t>0$ было построено семейство кубов $Q_{j}$ с попарно не пересекающимися внутренностями, обладающее следующими свойствами:

(a) $t<\left|Q_{j}\right|^{-1} \int_{Q_{j}}|f| \leqslant 2^{n} t$;

(b) $|f| \leqslant t$ п.в. вне множества $\bigcup_{j} Q_{j}$.

(Случай, когда такое семейство оказывается пустым, не исключен.) Конструкция с тех пор неоднократно воспроизводилась в учебниках, и мы не приводим ее.

С семейством $\left\{Q_{j}\right\}$ мы следующим образом свяжем усредняющий оператор $P_{t}=P_{t}^{f}$ :

$$
P_{t}^{f} h=\sum_{j} \frac{1}{\int \psi_{j}}\left(\int h \psi_{j}\right) \psi_{j}+h \psi_{\infty}
$$

где $\psi_{j}$ - характеристическая функция куба $Q_{j}$, а $\psi_{\infty}$ - характеристическая функция множества $\mathbb{R}^{n} \backslash \bigcup_{j} Q_{j}$. Коэффициенты в первой сумме - это просто средние $\left|Q_{j}\right|^{-1} \int_{Q_{j}} h$; мы записали их в иной форме для большего сходства с формулами $\S 2$.

Положим $f_{t}=P_{t}^{f} f=\sum c_{j} \psi_{j}+\psi_{\infty} f$, где $c_{j}=\left|Q_{j}\right|^{-1} \int_{Q_{j}} f$ - соответствующие средние функции $f$. Тогда $f-f_{t}=\sum\left(f-c_{j}\right) \psi_{j} ; j$-е слагаемое в этой сумме сосредоточено на кубе $Q_{j}$ и имеет нулевой интеграл.

Нетрудно усмотреть, что

$$
\left\|f-f_{t}\right\|_{L^{1}} \leqslant 2^{n+2} \operatorname{dist}_{L^{1}}\left(f, B_{L^{\infty}}(t / 2)\right) .
$$

Действительно, прежде всего,

$$
\left\|f-f_{t}\right\|_{L^{1}} \leqslant \sum\left\|\left(f-c_{j}\right) \psi_{j}\right\|_{L^{1}} \leqslant 2 \sum_{j} \int_{Q_{j}}|f| \leqslant 2^{n+1} t \sum_{j}\left|Q_{j}\right|
$$

в силу свойства (а). Далее, если $g \in B_{L^{\infty}}(t / 2)$, то

$$
\|f-g\|_{L^{1}} \geqslant \int_{\cup Q_{j}}|f|-\int_{\cup Q_{j}}|g| \geqslant t \sum\left|Q_{j}\right|-\frac{t}{2} \sum\left|Q_{j}\right|=\frac{t}{2} \sum\left|Q_{j}\right|,
$$

так что неравенство (2) выполняется ввиду предыдущей оценки.

Так как $\left|f_{t}\right| \leqslant 2^{n} t$ п.в., мы видим, что конструкция Кальдерона-Зигмунда действительно дает элемент почти оптимального приближения в смысле первой формулы в (1), если взять там $X=L^{\infty}$.

1.2. Почти оптимальное приближение в паре $\left(L^{1}, L^{p}\right), 1<p<\infty$. Оказывается, здесь тоже годится классическая процедура Кальдерона-Зигмунда, надо только правильно выбрать параметр $t$. Похоже, это не было замечено 
ранее. Чтобы сформулировать результат, напомним определение $K$-функционала.

Пусть $\left(X_{0}, X_{1}\right)$ - банахова пара. $K$-функционал $K\left(x, \sigma ; X_{0}, X_{1}\right)$ этой пары задается для $x \in X_{0}+X_{1}$ и $\sigma>0$ формулой

$$
K\left(x, \sigma ; X_{0}, X_{1}\right)=\inf \left\{\left\|x_{0}\right\|_{X_{0}}+\sigma\left\|x_{1}\right\|_{X_{1}}: x_{0} \in X_{0}, x_{1} \in X_{1}, x_{0}+x_{1}=x\right\} .
$$

Tеорема 1. Пусть $f \in L^{1}\left(\mathbb{R}^{n}\right) u f \notin B_{L^{p}\left(\mathbb{R}^{n}\right)}(s)$. Положим

$$
t(s)=2 \lambda(s)^{-1} K\left(f, \lambda(s) ; L^{1}, L^{\infty}\right),
$$

где $\lambda(s)=\left(s^{-1} \operatorname{dist}_{L^{1}\left(\mathbb{R}^{n}\right)}\left(f, B_{L^{p}\left(\mathbb{R}^{n}\right)}(s)\right)\right)^{q}, q^{-1}+p^{-1}=1$. Тогда

$$
\left\|f_{t(s)}\right\|_{L^{p}\left(\mathbb{R}^{n}\right)} \leqslant C s \quad u \quad\left\|f-f_{t(s)}\right\|_{L^{1}\left(\mathbb{R}^{n}\right)} \leqslant C \operatorname{dist}_{L^{1}\left(\mathbb{R}^{n}\right)}\left(f, B_{L^{p}\left(\mathbb{R}^{n}\right)}(s)\right),
$$

где $C$ не зависит от $s$ и $f$. Более того, для кубов $Q_{i}$ Кальдерона-Зигмунда, входящих в определение функции $f_{t(s)}$, верна оценка

$$
\sum\left|Q_{i}\right| \leqslant C \lambda(s)
$$

Доказательство довольно громоздко, хоть и элементарно; его можно найти в препринте [12].

1.3. Сингулярные интегральные операторы. Известно несколько похожих, но не вполне одинаковых определений этого понятия. Мы не воспроизводим ни одно из них, а просто перечислим свойства операторов, нужные в доказательствах (в $§ 2$ список этих свойств будет несколько расширен).

Пусть $p \in(1, \infty)$. Обозначим через $S_{p}$ класс линейных операторов $T$, удовлетворяющих следующим условиям:

(A) $T$ непрерывно отображает пространство $L^{p}\left(\mathbb{R}^{n}\right)$ в себя;

(В) $T$ непрерывно отображает пространство $L^{1}\left(\mathbb{R}^{n}\right)$ в пространство измеримых функций со сходимостью по мере;

(C) если $Q-$ куб, а функция $g \in L^{1}\left(\mathbb{R}^{n}\right)$ имеет нулевое среднее и сосредоточена на $Q$, то $\int_{\mathbb{R}^{n} \backslash(2 Q)}|T g| \leqslant C \int_{\mathbb{R}^{n}}|g|$ ( $\alpha Q$ всюду означает куб диаметра $\alpha \operatorname{diam} Q$ с тем же центром; постоянная $C$ не зависит от $g$ и $Q$ ).

Хорошо известно, что всякий сингулярный интегральный оператор типа Кальдерона-Зигмунда лежит в $S_{p}$ при $1<p<\infty$, см., например, [14], [15]. Заметим, однако, что если $T \in S_{p}$ и $h \in L^{\infty}\left(\mathbb{R}^{n}\right)$, то оператор $T_{1}, T_{1} f=h T f$, тоже лежит в $S_{p}$. Мы будем пользоваться этим наблюдением в случае, когда $h$ - характеристическая функция измеримого множества.

1.4. Результаты. Все, наконец, готово, и мы приводим формулировку.

Теорема 2. Пусть $p \in(1, \infty)$ u $T \in S_{p}$. Предположим, что $f \in L^{1}\left(\mathbb{R}^{n}\right)$, $s>0$ и $T f \in L^{1}\left(\mathbb{R}^{n}\right)$. Тогда найдется функиия $g \in B_{L^{p}}(c s)$, такая, что

$$
\begin{gathered}
\|f-g\|_{L^{1}} \leqslant C \operatorname{dist}_{L^{1}}\left(f, B_{L^{p}}(s)\right), \\
\|T f-T g\|_{L^{1}} \leqslant C\left(\operatorname{dist}_{L^{1}}\left(f, B_{L^{p}}(s)\right)+\operatorname{dist}_{L^{1}}\left(T f, B_{L^{p}}(s)\right)\right) .
\end{gathered}
$$

При этом функцию $g$ можно указать явно с помощъю процедуры КальдеронаЗигмунда: если $f \notin B_{L^{p}}(s)$, то достаточно положить $g=f_{t(s)}$, где число $t(s)$ определено формулой (3). Постоянные с и $C$ не зависят от участвующих функиий и от $s$.

Разумеется, если $f \in B_{L^{p}}(s)$, достаточно просто взять $g=f$. 
Из последнего абзаца предыдущего раздела немедленно вытекает следующий «локальный» вариант теоремы 2.

СлеДСтвие 1. Пусть $p \in(1, \infty)$ u $T \in S_{p}$. Предположим, ито $f \in L^{1}\left(\mathbb{R}^{n}\right)$, $s>0$, а измеримое множество $E$ таково, что $\chi_{E} T f \in L^{1}(E)$. Тогда функиия $g$, указанная в теореме 2 , удовлетворяет соотношениям $\|f-g\|_{L^{1}} \leqslant$ $C \operatorname{dist}_{L^{1}}\left(f, B_{L^{p}}(s)\right) u$

$$
\int_{E}|T f-T g| \leqslant C\left(\operatorname{dist}_{L^{1}}\left(f, B_{L^{p}}(s)\right)+\operatorname{dist}_{L^{1}(E)}\left(\chi_{E} T f, B_{L^{p}(E)}(s)\right)\right) .
$$

ДОКАЗАТЕЛЬСТВо ТЕОРЕМЫ 2. ВЫчисления навеяны статьей [4], однако имеется отличие, о котором мы скажем ниже.

Можно считать, что $f \notin B_{L^{p}}(s)$. Тогда положим $g=f_{t(s)}$ и выберем функцию $u \in B_{L^{p}}(s)$, удовлетворяющую неравенству

$$
\|T f-u\|_{L^{1}} \leqslant 2 \operatorname{dist}_{L^{1}}\left(T f, B_{L^{p}}(s)\right) .
$$

Имеем

$$
\|T f-T g\|_{L^{1}} \leqslant \int_{\mathbb{R}^{n} \backslash\left(\cup 2 Q_{i}\right)}|T f-T g|+\int_{\bigcup\left(2 Q_{i}\right)}|T f-u|+\int_{\bigcup\left(2 Q_{i}\right)}|T g-u|,
$$

где $\left\{Q_{i}\right\}$ - кубы, возникающие при разбиении функции $f$ по Кальдерону и Зигмунду с параметром $t=t(s)$ (см. разд. 1.1-1.2). Средний член справа оценивается с помощью неравенства (5). Так как $f-g=f-f_{t(s)}=\sum\left(f-c_{i}\right) \chi_{Q_{i}}$ и слагаемые имеют нулевые средние, то, благодаря свойству (C) операторов класса $S_{p}$, мы можем оценить первый член так:

$$
\begin{aligned}
\int_{\mathbb{R}^{n} \backslash\left(\cup 2 Q_{i}\right)}|T f-T g| & \leqslant \sum_{i} \int_{\mathbb{R}^{n} \backslash\left(2 Q_{i}\right)}\left|T\left(\left(f-c_{i}\right) \chi_{Q_{i}}\right)\right| \leqslant C \sum_{i} \int_{Q_{i}}\left|f-c_{i}\right| \\
& \leqslant C\|f-g\|_{L^{1}} \leqslant C^{\prime} \operatorname{dist}_{L^{1}}\left(f, B_{L^{p}}(s)\right) .
\end{aligned}
$$

Мы воспользовались тем, что $g$ совпадает с $c_{i}$ на $Q_{i}$, при третьем переходе и теоремой 1 при четвертом. K последнему слагаемому справа в (6) применим неравенство Гёльдера $\left(q^{-1}+p^{-1}=1\right)$ :

$$
\int_{\bigcup\left(2 Q_{i}\right)}|T g-u| \leqslant\left|\bigcup 2 Q_{i}\right|^{1 / q}\left(\|T g\|_{L^{p}}+\|u\|_{L^{p}}\right) \leqslant C s\left(\sum\left|Q_{i}\right|\right)^{1 / q} .
$$

Мы воспользовались здесь тем, что $u \in B_{L^{p}}(s)$, а также тем, что $\|T g\|_{L^{p}} \leqslant$ $C\|g\|_{L^{p}} \leqslant C^{\prime} s$ по теореме 1 . В силу неравенства (4) получаем окончательно $\int_{\bigcup\left(2 Q_{i}\right)}|T g-u| \leqslant C^{\prime \prime} \operatorname{dist}_{L^{1}}\left(f, B_{L^{p}}(s)\right)$. Осталось собрать оценки вместе.

ЗАмечАние. Рассуждения Бургейна [4] диктуют иной выбор функции $g$, а именно $g=h+P_{t}^{(f-h)}(f-h)$, где $h-$ произвольная функция, удовлетворяющая условиям $h \in B_{L^{p}}(s),\|f-h\|_{L^{1}} \leqslant 2 \operatorname{dist}_{L^{1}}\left(f, B_{L^{p}}(s)\right)$ (последнее расстояние снова предполагается положительным). Параметр $t$ на сей раз определяется из уравнения $t^{1 / q}\|f-h\|_{L^{1}}^{1 / q}=s$. Вычисление, показывающее, что и такая функция $g$ годится, слегка отличается от приведенного выше.

На этом пути не нужна довольно громоздкая теорема 1, однако некоторым недостатком (психологическим?) является то, что разложению КальдеронаЗигмунда подвергается «неизвестная» функция $f-h$ вместо «известной» функции $f$. 


\section{5. Приложения.}

СледСтвие 2. Предположим, что $T \in S_{p}, f \in L^{1}$ и функиия $T f$ интегрируема на множестве $E \subset \mathbb{R}^{n}$. Тогда существует последовательность $f_{k} \in L^{1} \cap L^{p}$, такая, что $f_{k} \rightarrow f$ в $L^{1}$, все функиии $T f_{k}$ интегрируемын н $E$ u $\int_{E}\left|T f_{k}-T f\right| \rightarrow 0$.

ДокАЗАТЕЛЬСтво. Заметим, что функция $f$ лежит в замыкании пространства $L^{1} \cap L^{p}$, а функция $\chi_{E} T f-$ в замыкании пространства $L^{1}(E) \cap L^{p}(E)$, после чего применим следствие 1.

Следующая лемма будет использоваться в $§ 2$.

Лемма 1. Предположим, что $T \in S_{p}$, a $E$ - измеримое подмножсество в $\mathbb{R}^{n}$. Пусть функиия $\psi \in L^{\infty} \cap L^{q}\left(q^{-1}+p^{-1}=1\right)$ сосредоточена на $E$ и такова, что $T^{*} \psi \in L^{\infty}$. Если функиия $T f$ суммируема на $E$, mо $\int_{E} \psi T f=\int_{\mathbb{R}^{n}}\left(T^{*} \psi\right) f$.

ДоказАТЕЛЬСтво. Возьмем функции $f_{k}$, как в следствии 2 , и напишем

$$
\int_{E} \psi T f=\lim _{k} \int_{\mathbb{R}^{n}} \psi T f_{k}=\lim _{k} \int_{\mathbb{R}^{n}}\left(T^{*} \psi\right) f_{k}=\int_{\mathbb{R}^{n}}\left(T^{*} \psi\right) f .
$$

Переход к сопряженному оператору во втором равенстве - законная операция, так как оператор $T$ ограничен в $L^{p}, f_{k} \in L^{p}$, а $\psi \in L^{q}$.

\section{§2. Классы Липшица}

2.1. Аналог разложения Кальдерона-Зигмунда. Мы работаем с однородными липшицевыми классами $\operatorname{Lip}_{\alpha}\left(\mathbb{R}^{n}\right), 0<\alpha<1$, снабженными нормой (в действительности полунормой, обращающейся в нуль на константах) $\|f\|_{\operatorname{Lip}_{\alpha}}=\sup _{x \neq y}|x-y|^{-\alpha}|f(x)-f(y)|$. Аналог разложения Кальдерона-Зигмунда для пары $\left(L^{1}\left(\mathbb{R}^{n}\right), \operatorname{Lip}_{\alpha}\left(\mathbb{R}^{n}\right)\right)$ был построен в диссертации [10]. Сформулируем этот результат. Его доказательство мы обсудим в приложении в конце статьи. Под укладкой понимается набор кубов с непересекающимися внутренностями.

Пусть $f \in L^{1}\left(\mathbb{R}^{n}\right), t>0$ и $f \notin B_{\operatorname{Lip}_{\alpha}}(t)$ (последнее условие наложено для того, чтобы избежать вырождений). Процедура, о которой только что говорилось, приводит к набору кубов $\left\{Q_{i}\right\}$ и набору неотрицательных $C^{\infty}$-функций $\left\{\psi_{i}\right\}$, $\operatorname{supp} \psi_{i} \subset \frac{1}{2} Q_{i}$, обладающих следующими свойствами.

Кубы. (I) $\bigcup\left(\frac{1}{2} Q_{i}\right)=\mathbb{R}^{n}$.

(II) Набор $\left\{Q_{i}\right\}$ разбивается на не более, чем $B_{n}$, укладок, где число $B_{n}$ зависит лишь от размерности $n$.

(III) Если $Q_{i} \cap Q_{j} \neq \varnothing$, то $\left|Q_{i} \cap Q_{j}\right| \geqslant \varepsilon(n) \max \left\{\left|Q_{i}\right|,\left|Q_{j}\right|\right\}$, где число $\varepsilon(n)$ опять зависит только от $n$.

Из II и III легко вывести еще одно условие:

(IV) $\operatorname{card}\left\{i: Q_{i} \cap Q_{j} \neq \varnothing\right\} \leqslant \gamma(n)$ при всех $j$, где $\gamma(n)$ зависит только от $n$.

Действительно, в силу (III) в любой укладке, составленной из кубов набора $\left\{Q_{i}\right\}$, не более $\varepsilon(n)^{-1}$ кубов, отличных от $Q_{j}$, пересекают $Q_{j}$.

Функции $\boldsymbol{\psi}_{\boldsymbol{i}}$. Набор функций $\left\{\psi_{i}\right\}$ представляет собой разбиение единицы на $\mathbb{R}^{n}$. Уже упоминалось, что $\operatorname{supp} \psi_{i} \subset \frac{1}{2} Q_{i}$. Кроме того, на размер этих функций накладывается следующее условие:

$$
\left|\nabla \psi_{i}\right| \leqslant C\left|Q_{i}\right|^{-1 / n}, \quad \int \psi_{i} \geqslant c\left|Q_{i}\right|
$$


Определим на локально интегрируемых функциях $h$ усредняющий (или, скорее, сглаживающий) оператор $P_{t}=P_{t}^{f}$ следующим образом (ср. с разд. 1.1):

$$
P_{t}^{f} h=\sum\left(\frac{1}{\int \psi_{i}} \int h \psi_{i}\right) \psi_{i}
$$

Из построения функций $\psi_{i}$ и кубов $Q_{i}$ вытекают следующие свойства:

$$
\begin{gathered}
\left\|P_{t}^{f} f\right\|_{\operatorname{Lip}_{\alpha}} \leqslant c t \\
\sum\left|Q_{i}\right|^{1+\alpha / n} \leqslant \frac{c}{t} \operatorname{dist}_{L^{1}}\left(f, B_{\operatorname{Lip}_{\alpha}}(t)\right) .
\end{gathered}
$$

Далее, положим $c_{i}=\left(\int \psi_{i}\right)^{-1} \int f \psi_{i}$ (так что $\left.P_{t}^{f} f=\sum c_{i} \psi_{i}\right)$. Выполнено еще одно условие:

$$
\left|Q_{i}\right|^{-1-\alpha / n} \int_{Q_{i}}\left|f-c_{i}\right| \leqslant c t .
$$

Из (CZ3) и (CZ4) легко вытекает, что

$$
\left\|f-P_{t}^{f} f\right\|_{L^{1}} \leqslant C \operatorname{dist}_{L^{1}}\left(f, B_{\operatorname{Lip}_{\alpha}}(t)\right) .
$$

Действительно,

$$
\left\|f-P_{t}^{f} f\right\|_{L^{1}} \leqslant \sum \int_{Q_{i}}\left|f-c_{i}\right| \leqslant C t \sum\left|Q_{i}\right|^{1+\alpha / n} \leqslant C \operatorname{dist}_{L^{1}}\left(f, B_{\operatorname{Lip}_{\alpha}}(t)\right) .
$$

Все оценочные постоянные зависят лишь от размерности $n$.

2.2. Оператор $\boldsymbol{T}$. Мы по-прежнему считаем, что «сингулярный» оператор $T$ лежит в $S_{p}$ хотя бы при одном $p \in(1, \infty)$. Еще нам понадобится, чтобы $T$ и $T^{*}$ переводили пространство $\operatorname{Lip}_{\alpha}$ в себя. Последнее действительно так, если $T$ - сингулярный интегральный оператор сверточного типа. По поводу не обязательно сверточных сингулярных интегралов и условий их действия в липшицевых классах см. [15, v. II].

Ограниченность оператора $T^{*}$ в $\operatorname{Lip}_{\alpha}$ будет нужна лишь для доказательства следующей леммы, которое мы отложим до разд. 2.4. В нем хватило бы ограниченности оператора $T^{*}$ в $\operatorname{Lip}_{\beta}$ при $\beta$, не обязательно совпадающем с $\alpha$.

ЛЕмма 2. Пусть T-оператор, подчиненный описанным условиям, и пусть $\psi$ есть $C^{\infty}$-функиия, сосредоточенная на кубе $Q$ и такал, что $|\psi| \leqslant A,|\nabla \psi| \leqslant$ $A|Q|^{-1 / n}$. Тогда $\left|T^{*} \psi\right| \leqslant C(A, T, n)$.

\section{3. Основной результат.}

Tеорема 3. Пусть оператор $T$ maкой, как в разд. 2.2, и nусть $f$ u $T f$ лежат в $L^{1}\left(\mathbb{R}^{n}\right)$. Тогда для каждого $t>0$ существует функиия $g \in B_{\operatorname{Lip}_{\alpha}}(c t)$, такая, что $\|f-g\|_{L^{1}} \leqslant \operatorname{dist}_{L^{1}}\left(f, B_{\operatorname{Lip}_{\alpha}}(t)\right) u$

$$
\|T f-T g\|_{L^{1}} \leqslant C\left(\operatorname{dist}_{L^{1}}\left(f, B_{\operatorname{Lip}_{\alpha}}(t)\right)+\operatorname{dist}_{L^{1}}\left(T f, B_{\operatorname{Lip}_{\alpha}}(t)\right)\right) .
$$

Более того, если $f \in B_{\operatorname{Lip}_{\alpha}}(t)$, можно положить $g=f$, а в противном случае $g=P_{t}^{f} f$. Заметим также, что при сделанных предположениях $\mathrm{Tg} \in$ $B_{\text {Lip }_{\alpha}}\left(c^{\prime} t\right)$. Постоянные $c$ и $c^{\prime}$ не зависят от участвующих функций и от $t$. 
ДокаЗАтельСтво. Считаем, что $f \notin B_{\operatorname{Lip}_{\alpha}}(t)$, и положим $g=P_{t}^{f} f$. Выберем функцию $u$ из шара $B_{\operatorname{Lip}_{\alpha}}(t)$, приблизительно реализующую $L^{1}$-расстояние от функции $T f$ до этого шара, и запишем

$$
\begin{aligned}
\|T f-T g\|_{L^{1}} \leqslant\left\|P_{t}^{f} T(f-g)\right\|_{L^{1}}+\left\|\left(I-P_{t}^{f}\right) T(f-g)\right\|_{L^{1}} \\
\leqslant\left\|P_{t}^{f} T\left(I-P_{t}^{f}\right) f\right\|_{L^{1}}+\left\|\left(I-P_{t}^{f}\right)(T f-u)\right\|_{L^{1}} \\
\quad+\left\|\left(I-P_{t}^{f}\right) u\right\|_{L^{1}}+\left\|\left(I-P_{t}^{f}\right) T P_{t}^{f} f\right\|_{L^{1}} .
\end{aligned}
$$

2.3.1. Оценка первого слагаемого. Мы проверим, что $\left\|P_{t}^{f} T\left(I-P_{t}^{f}\right) f\right\|_{L^{1}} \leqslant$ $C \operatorname{dist}_{L^{1}}\left(f, B_{\operatorname{Lip}_{\alpha}}(t)\right)$. Именно в этом рассуждении содержатся главные технические нововведения. Поскольку функция $f$ не будет меняться, для краткости будем писать $P_{t}$ вместо $P_{t}^{f}$. Напомним также обозначение $c_{i}=\left(\int \psi_{i}\right)^{-1} \int f \psi_{i}$ из разд. 2.1.

Оператор $T$, если рассматривать его на $L^{p}, 1 \leqslant p \leqslant \infty$, переводит функции в функции, а если рассматривать его на $\operatorname{Lip}_{\alpha},-$ функции по модулю констант в функции по модулю констант, так что всякий раз в формулах нужна правильная интерпретация. Функция $\rho=T f-T g=T f-T P_{t} f$ локально суммируема, ибо функция $P_{t} f$ липшицева (см. (CZ2)), а значит, такова же и функция $T P_{t} f$. $\mathrm{C}$ другой стороны, $\rho$ действительно можно понимать как функцию, а не как класс по модулю констант, ибо $f-g \in L^{1}$ (см. (CZ5)). Таким образом, выражение $P_{t} \rho$ ( $L^{1}$-норму которого мы оцениваем) действительно имеет смысл.

Приступим к оценке. Имеем

$$
P_{t}\left(T\left(f-P_{t} f\right)\right)=\sum_{j}\left(\frac{1}{\int \psi_{j}} \int \psi_{j} T\left(f-P_{t} f\right)\right) \psi_{j} .
$$

Зафиксируем $j$ и оценим отдельно $j$-е слагаемое в последней сумме. Для этого запишем функцию $f-P_{t} f$ следующим образом:

$$
f-P_{t} f=\sum_{i: Q_{i} \cap Q_{j} \neq \varnothing}\left(f-c_{i}\right) \psi_{i}+\sum_{i: Q_{i} \cap Q_{j}=\varnothing}\left(f-c_{i}\right) \psi_{i} \stackrel{\text { def }}{=} u_{j}+v_{j} .
$$

Сумма, определяющая функцию $u_{j}$, конечна в силу свойства (IV) из разд. 2.1, а ряд, определяющий функцию $v_{j}$, сходится в $L^{1} ;$ поэтому

$$
T v_{j}=\sum_{i: Q_{i} \cap Q_{j}=\varnothing} T\left(\left(f-c_{i}\right) \psi_{i}\right)
$$

в смысле сходимости по мере (разд. 1.3 , условие (В)). Рассмотрим измеримую функцию

$$
V_{j}=\sum_{i: Q_{i} \cap Q_{j}=\varnothing}\left|T\left(\left(f-c_{i}\right) \psi_{i}\right)\right|
$$

(возможно, она не является п.в. конечной). Тогда

$$
\sum_{j} \int_{Q_{j}} V_{j}=\sum_{i} \sum_{j: Q_{i} \cap Q_{j}=\varnothing} \int_{Q_{j}}\left|T\left(\left(f-c_{i}\right) \psi_{i}\right)\right| \leqslant C \sum_{i} \int_{\mathbb{R}^{n} \backslash Q_{i}}\left|T\left(\left(f-c_{i}\right) \psi_{i}\right)\right|,
$$

поскольку покрытие пространства $\mathbb{R}^{n}$ кубами $Q_{l}$ конечнократно (см. разд. 2.1, свойство (II)). Так как $\operatorname{supp} \psi_{i} \subset \frac{1}{2} Q_{i}$ (разд. 2.1), из условия (C) в разд. 1.3 
вытекает, что

$$
\sum_{j} \int_{Q_{j}} V_{j} \leqslant C \sum_{i} \int_{Q_{i}}\left|f-c_{i}\right| .
$$

Так как $\left|T v_{j}\right| \leqslant V_{j}$, отсюда следует, что функция $T v_{j}$ интегрируема на кубе $Q_{j}$ и что

$$
\sum_{j} \int_{Q_{j}}\left|T v_{j}\right| \leqslant C \sum_{i} \int_{Q_{i}}\left|f-c_{i}\right| .
$$

Далее, функция $T u_{j}=T\left(f-P_{t} f\right)-T v_{j}$ тоже интегрируема на кубе $Q_{j}$ (уже отмечалось, что функция $T\left(f-P_{t} f\right)$ локально интегрируема). По лемме 2 функции $T^{*} \psi_{j}$ равномерно ограничены. Применив лемму 1 , получим

$$
\int_{Q_{j}} \psi_{j} T u_{j}=\int\left(T^{*} \psi_{j}\right) u_{j}=\sum_{i: Q_{i} \cap Q_{j} \neq \varnothing} \int\left(T^{*} \psi_{j}\right)\left(f-c_{i}\right) \psi_{i}
$$

откуда

$$
\sum_{j}\left|\int_{Q_{j}} \psi_{j} T u_{j}\right| \leqslant C \sum_{i} \sum_{j: Q_{i} \cap Q_{j} \neq \varnothing} \int_{Q_{i}}\left|f-c_{i}\right| \leqslant C \gamma(n) \sum_{i} \int_{Q_{i}}\left|f-c_{i}\right|
$$

(см. разд. 2.1, свойство (IV)). Теперь напишем

$$
\begin{aligned}
\left|P_{t} T\left(f-P_{t} f\right)\right| & =\left|\sum_{j}\left[\frac{1}{\int \psi_{j}} \int\left(T u_{j}+T v_{j}\right) \psi_{j}\right] \psi_{j}\right| \\
& \leqslant \sum_{j} \frac{\psi_{j}}{\int \psi_{j}}\left(\left|\int_{Q_{j}} \psi_{j} T u_{j}\right|+\int_{Q_{j}}\left|T v_{j}\right|\right) .
\end{aligned}
$$

Проинтегрировав по $\mathbb{R}^{n}$ и учтя неравенства (8) и (9), получим

$$
\int\left|P_{t} T\left(I-P_{t}\right) f\right| \leqslant C \sum_{i} \int_{Q_{i}}\left|f-c_{i}\right| \leqslant C^{\prime} \operatorname{dist}_{L^{1}}\left(f, B_{\operatorname{Lip}_{\alpha}}(t)\right)
$$

(см. строку, доказывающую свойство (CZ5) в разд. 2.1).

2.3.2. Оценки остальных трех слагаемых в последней части неравенства (7) вполне рутинны.

$1^{\circ}$. Разумеется, $\left\|P_{t}\right\|_{L^{1} \rightarrow L^{1}} \leqslant C$ (получается непосредственно с использованием конечной кратности покрытия кубами $Q_{i}$ ). Поэтому второе слагаемое оценивается так:

$$
\left\|\left(I-P_{t}\right)(T f-u)\right\|_{L^{1}} \leqslant C\|T f-u\|_{L^{1}} \leqslant C^{\prime} \operatorname{dist}_{L^{1}}\left(T f, B_{\operatorname{Lip}_{\alpha}}(t)\right)
$$

в силу выбора функции $u$.

$2^{\circ}$. Проверим, что

$$
\left\|I-P_{t}\right\|_{\operatorname{Lip}_{\alpha} \rightarrow L^{1}} \leqslant C \frac{\operatorname{dist}_{L^{1}}\left(f, B_{\operatorname{Lip}_{\alpha}}(t)\right)}{t}
$$

(оператор $I-P_{t}$ обращается в нуль на константах; поэтому он переводит классы по модулю констант в настоящие функции, и все корректно). Если это неравенство доказано, то, заметив, что функции $u$ (см. третье слагаемое в (7)) и $T P_{t} f$ (четвертое слагаемое) обе лежат в шаре радиуса примерно $t$ в $\operatorname{Lip}_{\alpha}$ (см., среди 
прочего, неравенство (CZ2) в разд. 2.1), получаем, что оба слагаемых не превосходят величины $C \operatorname{dist}_{L^{1}}\left(f, B_{\operatorname{Lip}_{\alpha}}(t)\right)$. Чтобы завершить доказательство теоремы 3, остается собрать оценки всех четырех слагаемых вместе и вспомнить o (CZ5).

Итак, осталось доказать неравенство (10). Пусть $h \in \operatorname{Lip}_{\alpha}$. Надо оценить $L^{1}$-норму функции $\left(I-P_{t}\right) h$. Обозначив через $h_{Q}$ среднее функции $h$ по кубу $Q$, заметим, что

$$
\int_{Q}\left|h-h_{Q}\right| \leqslant C\|h\|_{\operatorname{Lip}_{\alpha}}|Q|^{1+\alpha / n} .
$$

Теперь мы можем написать

$$
\begin{aligned}
& \left\|\left(I-P_{t}\right) h\right\|_{L^{1}}=\int\left|h(x)-\sum_{i} \frac{1}{\int \psi_{i}} \int h(y) \psi_{i}(y) d y \psi_{i}(x)\right| d x \\
& \leqslant \sum_{i} \frac{1}{\int \psi_{i}} \iint|h(x)-h(y)| \psi_{i}(x) \psi_{i}(y) d x d y \\
& \leqslant \sum_{i} \frac{1}{\int \psi_{i}} \iint\left|h(x)-h_{Q_{i}}\right| \psi_{i}(x) \psi_{i}(y) d x d y \\
& +\sum_{i} \frac{1}{\int \psi_{i}} \iint\left|h(y)-h_{Q_{i}}\right| \psi_{i}(x) \psi_{i}(y) d x d y \\
& \leqslant C\|h\|_{\operatorname{Lip}_{\alpha}} \sum\left|Q_{i}\right|^{1+\alpha / n} \leqslant \frac{C^{\prime}}{t} \operatorname{dist}_{L^{1}}\left(f, B_{\operatorname{Lip}_{\alpha}}(t)\right)\|h\|_{\operatorname{Lip}_{\alpha}}
\end{aligned}
$$

в силу (СZ3).

2.4. Осталось еще привести пропущенное доказательство леммы 2. Докажем сначала неравенство

$$
\|g\|_{L^{\infty}} \leqslant C_{n, \alpha}\|g\|_{L^{q}}^{\alpha q /(n+\alpha q)}\|g\|_{\operatorname{Lip}_{\alpha}}^{n /(n+\alpha q)}
$$

где $\alpha \in(0,1], q \in[1, \infty)$, а функция $g$ такова, что правая часть имеет смысл. Пусть $\tau>0$, и предположим, что $|g(x)|>\tau$ для некоторого $x$. Из неравенства $|g(y)| \geqslant|g(x)|-b|x-y|^{\alpha}$, верного при всех $y$, видно, что множество $e=\{y$ : $|g(y)|>\tau / 2\}$ содержит шар $B=\left\{y:|x-y| \leqslant 2^{-1 / \alpha}(\tau / b)^{1 / \alpha}\right\}$. Следовательно, $|e| \geqslant|B| \geqslant C(\tau / b)^{n / \alpha}$. Так как $|e| \leqslant(2 / \tau)^{q} a^{q}$, получаем $(2 / \tau)^{q} a^{q} \geqslant C(\tau / b)^{n / \alpha}$, откуда $\tau \leqslant C^{\prime} a^{\alpha q /(n+\alpha q)} b^{n /(n+\alpha q)}$, а это и есть (12).

Теперь возьмем функцию $\psi$, как в лемме. Упомянутый в лемме оператор $T$ принадлежит классу $S_{p}$, в частности, ограничен в $L^{p}\left(\mathbb{R}^{n}\right)$. Поэтому оператор $T^{*}$ ограничен в $L^{q}\left(\mathbb{R}^{n}\right)$ при $q^{-1}+p^{-1}=1$. Ограничен он и в $\operatorname{Lip}_{\alpha}\left(\mathbb{R}^{n}\right)$ по условию. Неравенство (12) показывает, что

$$
\left\|T^{*} \psi\right\|_{L^{\infty}} \leqslant C\left\|T^{*} \psi\right\|_{L^{q}}^{\alpha q /(n+\alpha q)}\left\|T^{*} \psi\right\|_{\operatorname{Lip}_{\alpha}}^{n /(n+\alpha q)} \leqslant C^{\prime}\|\psi\|_{L^{q}}^{\alpha q /(n+\alpha q)}\|\psi\|_{\operatorname{Lip}_{\alpha}}^{n /(n+\alpha q)} .
$$

Однако $\|\psi\|_{L^{q}} \leqslant A|Q|^{1 / q}$, а для всяких $x, y \in Q$ верны оценки

$$
|\psi(x)-\psi(y)| \leqslant A|Q|^{-1 / n}|x-y| \leqslant A^{\prime}|Q|^{-1 / n}|Q|^{(1-\alpha) / n}|x-y|^{\alpha},
$$

т. е. $\|\psi\|_{\text {Lip }_{\alpha}} \leqslant A^{\prime}|Q|^{-\alpha / n}$. Подставив это в полученную выше оценку, придем к нужному неравенству $\left\|T^{*} \psi\right\|_{L^{\infty}} \leqslant$ const. 


\section{§3. Заключительные замечания}

Методы этой статьи вряд ли можно назвать простыми (в особенности это касается §2), и естественно спросить (что многие и делали), нельзя ли придти к тем же результатам как-то иначе. В частности, нас спрашивали, нельзя ли использовать свертки и нельзя ли использовать разложения по вейвлетам. Обсудим эти два вопроса.

3.1. Из сказанного ниже следует, что при попытке прибегнуть к сверткам мы встретим большие препятствия. Для простоты ограничимся случаем окружности $\mathbb{T}$ с мерой Лебега. Пусть $X_{0}, X_{1}$ - два трансляционно инвариантных пространства функций на окружности, причем для определенности пусть $X_{1} \subset$ $X_{0} \subset L^{1}$. Для всяких ли $f \in X_{0}$ и $t>0$ функцию $g$, такую, что

$$
\|f-g\|_{X_{0}} \leqslant C \operatorname{dist}_{X_{0}}\left(f, B_{X_{1}}(t)\right), \quad g \in B_{X_{1}}(c t),
$$

можно выбрать в виде $g=f * \tau$, где $\tau$ - некоторое распределение? (Разумеется, константы не должны зависеть от $f$ и $t$, а распределению $\tau$ это позволено.)

Заметим, что если $\hat{f}(n)=0$, то $\hat{g}(n)=0$ для функции $g$ указанного вида. В частности, если ответ положителен, то пара $\left(X_{0}^{\Lambda}, X_{1}^{\Lambda}\right) K$-замкнута в $\left(X_{0}, X_{1}\right)$ для любого множества $\Lambda \subset \mathbb{Z}\left(X^{\Lambda} \stackrel{\text { def }}{=}\{f \in X: \hat{f}(n)=0\right.$ при $\left.n \notin \Lambda\}\right)$. Интуиция подсказывает, что, в общем, так быть не должно. Простейший пример, когда все и вправду не так, дается парой $X_{0}=L^{1}, X_{1}=L^{\infty}$. В качестве $\Lambda$ можно взять множество степеней двойки. Классические результаты о лакунарных рядах говорят, что после перехода к коэффициентам Фурье пространство $\left(L^{p}\right)^{\Lambda}$ отождествляется с $l^{2}$ при $p<\infty$ и с $l^{1}$ при $p=\infty$. До сих пор мы избегали интерполяционной теории, но здесь она позволяет объяснить, в чем противоречие, очень коротко: если бы $K$-замкнутость присутствовала, имело бы место равенство $\left(\left(L^{1}\right)^{\Lambda},\left(L^{\infty}\right)^{\Lambda}\right)_{1 / 2,2}=\left(L^{2}\right)^{\Lambda}$. Однако $\left(l^{2}, l^{1}\right)_{1 / 2,2} \neq l^{2}$.

На эту тему возможны вариации. Пространство $L^{\infty}$ можно заменить пространством $L^{s}$ с $s<\infty$, воспользовавшись существованием множеств типа $\Lambda_{p}$, но не $\Lambda_{p+\varepsilon}$ при $p>2$, см. [16]. Далее, пространства $L^{1}, L^{s}$ можно заменить классами Харди $H^{1}, H^{s}$ (что более или менее равносильно введению в игру сингулярного интеграла - проектора Рисса). Мы опускаем детали.

3.2. Вейвлеты. Для простоты пусть в этом разделе все происходит на прямой $\mathbb{R}$. Пусть $\left\{h_{k, j}\right\}$ - какая-нибудь ортогональная система вейвлетов с достаточно гладкой порождающей функцией (из которой все $\left\{h_{k, j}\right\}$ получаются сдвигами и растяжениями). Известно (см. [15]), что тогда эта система - безусловный базис в $L^{p}(\mathbb{R})$ при $p>1$ и базис в $L^{1}(\mathbb{R})$ (не безусловный, ибо таковых в этом пространстве нет вообще). В пространстве Lip $\alpha$ базисов нет из-за несепарабельности, однако система $\left\{h_{n, j}\right\}$ ведет себя почти как безусловный базис при $0<\alpha<1$ : операторы $f \mapsto \sum a_{i j}\left(f, h_{k, j}\right) h_{k, j}\left\|h_{k, j}\right\|_{L^{2}}^{-2}$ ограничены равномерно при $\left|a_{i j}\right| \leqslant 1$.

Пусть теперь $X$ - это либо $L^{p}(1<p<\infty)$, либо $\operatorname{Lip}_{\alpha}(0<\alpha<1)$, и пусть $f \in L^{1}$. Нет ли процедуры, позволяющей получить почти оптимальное $L^{1}$-приближение $g$ функции $f$ шаром пространства $X$ с помощью ее разложения по системе $\left\{h_{k, j}\right\}$ ?

Ответа мы не знаем. Если эта гипотетическая процедура зависит лишь от модулей коэффициентов разложения функции $f$ по вейвлетам, то неясно, как 
оценивать норму $\|f-g\|_{L^{1}}$, поскольку в $L^{1}$ базис $\left\{h_{k, j}\right\}-$ не безусловный. У нас возникло впечатление, что если это препятствие и можно обойти, то примерно на том же уровне сложности, что встречался в $\S 1,2$. Тем не менее справедлив следующий результат.

ПРЕДЛОЖЕНИЕ 1. Пусть $\left\{h_{k, j}\right\}$ - система достаточно гладких вейвлетов, а $X$ - это либо $L^{p}, 1<p<\infty$, либо $\operatorname{Lip}_{\alpha}, 0<\alpha<1$. Пусть $f \in L^{1} u$ $t>0$. Тогда существует функиия $g$ из шара $B_{X}(c t)$, такая, что $\|f-g\|_{L^{1}} \leqslant$ $C \operatorname{dist}_{L^{1}}\left(f, B_{X}(t)\right)$, со следующим дополнительньм условием: коэфбфициенть разложсения функиии $g$ по системе $\left\{h_{k j}\right\}$ могут быть отличны от нуля лишь на тех местах, на которых отличны от нуля коэфбициенты разложсения функиии $f$.

ДоказАтельство. Пусть $E$ - множество тех пар $(k, j)$, для которых $\left(f, h_{k, j}\right)$ $\neq 0$. Рассмотрим оператор $T$,

$$
T \varphi=\sum_{(k, j) \in E}\left(\varphi, h_{k, j}\right)\left\|h_{k, j}\right\|_{L^{2}}^{-2} h_{k, j} .
$$

Это проектор, оставляющий функцию $f$ на месте. С другой стороны, хорошо известно, что $T$ - сингулярный интегральный оператор типа КальдеронаЗигмунда, см. [15], [17]. Осталось применить к этому оператору $T$ теорему 2 при $X=L^{p}$ или теорему 3 при $X=\operatorname{Lip}_{\alpha}$.

Таким образом, система вейвлетов обладает тем самым свойством, которое оказалось невозможным для тригонометрической системы (разд. 3.1), однако мы не видим иного способа убедиться в этом, кроме как прибегнуть к теоремам $\S \S 1,2$. В качестве $X$ в предложении 1 можно еще взять иные пространства гладких функций, рассмотренные в [12], [13].

\section{Приложение. Как построить разбиение Кальдерона-Зигмунда для пары $\left(L^{1}, \operatorname{Lip}_{\alpha}\right)$ ?}

Похожее разбиение для соболевских классов вместо липшицевых было построено в [11]. Здесь мы остановимся на основных моментах конструкции для классов Липшица. Больше подробностей читатель найдет в приложении 2 к препринту [12].

Пусть $\left\{Q_{x}\right\}_{x \in \mathbb{R}^{n}}$ - семейство кубов, причем для каждого $x$ куб $Q_{x}$ имеет своим центром $x$ и не исключено вырождение (когда $Q_{x}=\{x\}$ ). Определим $\beta$-емкость $(\beta>0)$ этого семейства (обозначение: $\left.\left|\left\{Q_{x}\right\}\right|_{\beta}\right)$ как наименьшее число $C$, такое, что $\sum\left|Q_{x_{i}}\right|^{\beta} \leqslant C$ для всех укладок $\left\{Q_{x_{i}}\right\}$, составленных из кубов семейства.

Радиус $r(Q)$ куба $Q$ - это половина длины его стороны. Куб радиуса $r$ с центром в $x$ обозначается через $Q(x, r)$. Пусть $f \in L^{1}\left(\mathbb{R}^{n}\right)$. Рассмотрим (ср. (11)) непрерывную по $r$ функцию $\varphi_{x}(r)=|Q(x, r)|^{-1-\alpha / n} \int_{Q(x, r)}\left|f-f_{Q(x, r)}\right|$. Ясно, что $\lim _{r \rightarrow \infty} \varphi_{x}(r)=0$. Пусть $t>0$, а $b$ - постоянная, которая будет зафиксирована чуть позже. Введем семейство $\left\{Q_{x}\right\}_{x \in \mathbb{R}^{n}}$ nределъных кубов следующим образом: $Q_{x}=\{x\}$, если $\sup _{r>0} \varphi_{x}(r) \leqslant b t$, а в противном случае $Q_{x}=Q\left(x, r_{x}\right)$, где $r_{x}=\sup \left\{r: \varphi_{x}(t)>b t\right\}$. Оценим $(1+\alpha / n)$-емкость этого семейства. Возьмем произвольную функцию $g$ из $B_{\operatorname{Lip}_{\alpha}}(t)$. Пусть $\left\{K_{j}\right\}-$ укладка, составленная из предельных кубов. Учитывая неравенство $\int_{Q}|f-c| \geqslant 2^{-1} \int_{Q}\left|f-f_{Q}\right|$ (верное для 
любой константы $c$ ), определение предельного куба и формулу (11), получим

$$
\begin{aligned}
\|f-g\|_{L^{1}} & \geqslant \sum_{j} \int_{K_{j}}|f-g| \geqslant \sum_{j} \int_{K_{j}}\left|f-g_{K_{j}}\right|-\sum_{j} \int_{K_{j}}\left|g-g_{K_{j}}\right| \\
& \geqslant \sum_{j} 2^{-1} \int_{K_{j}}\left|f-f_{K_{j}}\right|-\sum_{j} \int_{K_{j}}\left|g-g_{K_{j}}\right| \\
& \geqslant \frac{b}{2} t \sum_{j}\left|K_{j}\right|^{1+\alpha / n}-C t \sum_{j}\left|K_{j}\right|^{1+\alpha / n}=\frac{b}{4} t \sum_{j}\left|K_{j}\right|^{1+\alpha / n},
\end{aligned}
$$

если с самого начала $b=4 C$. Таким образом,

$$
\left|\left\{Q_{x}\right\}\right|_{1+\alpha / n} \leqslant \frac{4}{b} t^{-1} \operatorname{dist}_{L^{1}}\left(f, B_{\operatorname{Lip}_{\alpha}}(t)\right) .
$$

Последняя оценка позволяет сделать главный шаг - применить теорему о покрытии из статьи [11]. В результате получится семейство кубов $\left\{Q_{i}\right\}$ со следующими свойствами. Прежде всего,

$$
\sum\left|Q_{i}\right|^{1+\alpha / n} \leqslant\left|\left\{Q_{x}\right\}\right|_{1+\alpha / n} \leqslant \frac{4}{b} t^{-1} \operatorname{dist}_{L^{1}}\left(f, B_{\operatorname{Lip}_{\alpha}}(t)\right) .
$$

(Это - свойство (CZ3) из разд. 2.1.) Далее, $Q_{x_{i}} \subset Q_{i}$, где $x_{i}$ - центр куба $Q_{i}$, и для всякого $x$ существует индекс $i(x)$, такой, что $Q_{x} \subset Q_{i(x)}$. Наконец, выполнены условия (II) и (III) из разд. 2.1 , а условие (I) выполнено в усиленной форме: $\bigcup\left(\frac{1}{4} Q_{i}\right)=\mathbb{R}^{n}$.

Функции $\psi_{i}$, упомянутые в разд. 2.1 , строятся обычным образом: возьмем $C^{\infty}$-функции $\varphi_{i}, 0 \leqslant \varphi_{i} \leqslant 1$, равные 1 на $\frac{1}{4} Q_{i}$ и 0 вне $\frac{1}{2} Q_{i}$, и положим $\psi_{i}=\varphi_{i}\left[\sum_{k} \varphi_{k}\right]^{-1}$. Оценку градиента в (CZ1), разд. 2.1, проще всего обеспечить, считая, что все функции $\varphi_{i}$ получаются растяжениями и сдвигами из одной. Из свойства (IV), разд. 2.1 , видно, что $\psi_{i} \geqslant d(n)$ на $\frac{1}{4} Q_{i}$; поэтому $\int_{Q_{i}} \psi_{i} \geqslant d^{\prime}(n)\left|Q_{i}\right|$ (второе неравенство в (CZ1)). Отсюда выводится условие (CZ4) из разд. 2.1:

$\int_{Q_{i}}\left|f-c_{i}\right| \leqslant \int_{Q_{i}} \int_{Q_{i}} \frac{1}{\int \psi_{i}}|f(x)-f(y)| d x d y \leqslant C\left|Q_{i}\right|^{-1} \int_{Q_{i}} \int_{Q_{i}}|f(x)-f(y)| d x d y$; далее нужно написать $|f(x)-f(y)| \leqslant\left|f(x)-f_{Q_{i}}\right|+\left|f(y)-f_{Q_{i}}\right|$ и воспользоваться тем, что $Q_{i}$ содержит предельный куб с тем же центром.

Осталось проверить условие (CZ2) из разд. 2.1. Будем писать $f_{t}$ вместо $P_{t}^{f} f$. Неравенство (11) в действительности характеризует класс $\operatorname{Lip}_{\alpha}$ (см., например, [18]); поэтому достаточно показать, что $|Q|^{-1-\alpha / n} \int_{Q}\left|f_{t}-\left(f_{t}\right)_{Q}\right| \leqslant C(n) t$ для всех кубов $Q$. Рассмотрим два случая.

Случай 1. $Q \subset Q_{i_{0}}$ для некоторого $i_{0}$. Легко видеть, что $\int_{Q}\left|f_{t}-\left(f_{t}\right)_{Q}\right| \leqslant$ $|Q|^{1+1 / n} \max _{Q}\left|\nabla f_{t}\right|$. Поэтому достаточно проверить неравенство $\max _{Q}\left|\nabla f_{t}\right| \leqslant$ $C t|Q|^{-(1-\alpha) / n}$. Установим более сильное неравенство

$$
\max _{Q_{i_{0}}}\left|\nabla f_{t}\right| \leqslant C t\left|Q_{i_{0}}\right|^{-(1-\alpha) / n} .
$$

Из равенства $\sum \psi_{i} \equiv 1$ получаем $\partial \psi_{i_{0}} / \partial x_{k}=-\sum_{i \neq i_{0}} \partial \psi_{i} / \partial x_{k}$. Так как $f_{t}=$ $\sum c_{i} \psi_{i}$, отсюда следует, что $\partial f_{t} / \partial x_{k}=\sum_{i}\left(c_{i}-c_{i_{0}}\right) \partial \psi_{i} / \partial x_{k}$. Лишь $\gamma(n)$ слагаемых в последней сумме могут быть отличны от нуля на $Q_{i_{0}}$ (см. свойство (IV) 
в разд. 2.1). Так как $\left|\nabla \psi_{i}\right| \leqslant C\left|Q_{i}\right|^{-1 / n}$, мы видим, что

$$
\max _{Q_{i_{0}}}\left|\nabla f_{t}\right| \leqslant C^{\prime} \max _{i: Q_{i} \cap Q_{i_{0}} \neq \varnothing}\left|c_{i}-c_{i_{0}}\right|\left|Q_{i}\right|^{-1 / n} \text {. }
$$

Но если $Q_{i} \cap Q_{i_{0}} \neq \varnothing$, то объемы множеств $Q_{i}, Q_{i_{0}}$ и $Q_{i} \cap Q_{i_{0}}$ сравнимы (см. свойство (III) в разд. 2.1). Поэтому

$$
\begin{aligned}
\max _{Q_{i_{0}}}\left|\nabla f_{t}\right| & \leqslant C^{\prime} \max _{i: Q_{i} \cap Q_{i_{0}} \neq \varnothing}\left|Q_{i_{0}} \cap Q_{i}\right|^{-1} \int_{Q_{i_{0}} \cap Q_{i}}\left|c_{i}-c_{i_{0}}\right|\left|Q_{i_{0}}\right|^{-1 / n} \\
& \leqslant C^{\prime \prime} \max _{i: Q_{i} \cap Q_{i_{0}} \neq \varnothing}\left|Q_{i_{0}}\right|^{-1-1 / n}\left(\int_{Q_{i}}\left|f-c_{i}\right|+\int_{Q_{i_{0}}}\left|f-c_{i_{0}}\right|\right) \\
& \leqslant C^{\prime \prime \prime} \max _{i: Q_{i} \cap Q_{i_{0}} \neq \varnothing}\left|Q_{i_{0}}\right|^{-1-1 / n} t\left(\left|Q_{i}\right|^{1+\alpha / n}+\left|Q_{i_{0}}\right|^{1+\alpha / n}\right) \\
& \leqslant C^{\prime \prime \prime \prime \prime} t\left|Q_{i_{0}}\right|^{(1-\alpha) / n}
\end{aligned}
$$

что и требовалось.

Cлучай 2. Куб $Q$ не содержится ни в одном из $Q_{i}$. Пусть $x$ - центр куба $Q$. Предельный куб $Q_{x}$ содержится в одном из кубов $Q_{i}$; поэтому $Q \not \subset Q_{x}$. А тогда $Q_{x} \subset Q$ и, следовательно, $|Q|^{-1-\alpha / n} \int_{Q}\left|f-f_{Q}\right| \leqslant b t$.

Докажем теперь, что $\sum_{Q_{i} \cap Q \neq \varnothing}\left|Q_{i}\right| \leqslant C(n)|Q|$. Так как набор $\left\{Q_{i}\right\}$ разбивается на $B(n)$ укладок, достаточно показать, что $Q_{i} \subset C(n) Q$, если $Q_{i} \cap Q \neq \varnothing$. Но кубы $\frac{1}{4} Q_{i}$ покрывают $\mathbb{R}^{n}$; поэтому всякая точка $y$ из $Q_{i} \cap Q$ содержится в $\frac{1}{4} Q_{i_{0}}$ при некотором $i_{0}=i_{0}(y)$. Однако $Q \not \subset Q_{i_{0}}$; поэтому $\frac{3}{4} r\left(Q_{i_{0}}\right) \leqslant 2 r(Q)$, откуда $r\left(Q_{i_{0}}\right) \leqslant 3 r(Q)$. Поскольку объемы кубов $Q_{i_{0}}$ и $Q_{i}$ сравнимы (разд. 2.1, свойство (III)), мы приходим к требуемому утверждению.

Остается написать

$$
\begin{aligned}
\int_{Q}\left|f_{t}-\left(f_{t}\right)_{Q}\right| & \leqslant 2 \int_{Q}\left|f_{t}-f_{Q}\right| \leqslant 2\left(\int_{Q}\left|f-f_{Q}\right|+\int_{Q}\left|f-f_{t}\right|\right) \\
& \leqslant 2 b t|Q|^{1+\alpha / n}+2 \sum_{Q_{i} \cap Q \neq \varnothing} \int_{Q_{i}}\left|f-c_{i}\right| \\
& \leqslant 2 b t|Q|^{1+\alpha / n}+C t \sum_{Q_{i} \cap Q \neq \varnothing}\left|Q_{i}\right|^{1+\alpha / n}
\end{aligned}
$$

и оценить последнюю сумму величиной $\left(\sum_{Q_{i} \cap Q \neq \varnothing}\left|Q_{i}\right|\right)^{1+\alpha / n}$, которая, как мы только что видели, не превосходит $C|Q|^{1+\alpha / n}$.

\section{ЛитерАТУРА}

[1] G. Pisier, "Interpolation between $H^{p}$-spaces and noncommutative generalizations. I", Pacific J. Math., 155:2 (1992), 341-368.

[2] G. Pisier, "Interpolation between $H^{p}$-spaces and noncommutative generalizations. II", Rev. Mat. Iberoamericana, 9:2 (1993), 281-291.

[3] S. V. Kislyakov, Quanhua Xu, "Interpolation of weighted and vector-valued Hardy spaces", Trans. Amer. Math. Soc., 343:1 (1994), 1-34.

[4] J. Bourgain, "Some consequences of Pisier's approach to interpolation", Israel J. Math., 77:1-2 (1992), 165-185. 
[5] С. В. Кисляков, Кванхуа Шу, "Вещественная интерполяция и сингулярные интегралы", Алгебра и анализ, 8:4 (1996), 75-109.

[6] S. V. Kislyakov, Quanhua Xu, "Partial retractions for weighted Hardy spaces", Studia Math., 138:3 (2000), 251-264.

[7] S. V. Kislyakov, "Interpolation of $H^{p}$-spaces: some recent developments", in: Function Spaces, Interpolation Spaces, and Related Topics (Haifa, 1995), Israel Math. Conf. Proc., vol. 13, Bar-Ilan Univ., Ramat Gan, 1999, 102-140.

[8] T. W. Gamelin, S. V. Kislyakov, "Uniform algebras as Banach spaces", in: Handbook of the Geometry of Banach Spaces, vol. 1, Elsevier, 2001, 671-706.

[9] A. P. Calderón, A. Zygmund, "On the existence of certain singular integrals", Acta Math., 88 (1952), 85-139.

[10] Н. Я. Кругляк, Исследование вещественного метода интерполяиии, Дисс. д.ф.м.н., 1996.

[11] Н. Я. Кругляк, “Гладкие аналоги разложения Кальдерона-Зигмунда, количественные теоремы о покрытиях и $K$-функционал для пары $\left(L_{q}, \dot{W}_{p}^{k}\right)$ ", Алгебра и анализ, 8:4 (1996), 110-160.

[12] S. V. Kislyakov, N. Ya. Kruglyak, Stability of approximation under singular integrals, and Calderón-Zygmund type decompositions, PDMI Preprint No. 7, 2005; www.pdmi.ras.ru/preprint/2005/index.html.

[13] S. V. Kislyakov, N. Ya. Kruglyak, Stability of approximation under singular integrals and Calderón-Zygmund type decompositions. II, Preprint of the Erwin Schrödinger Institute No. 1734, 2005; www.esi.ac.at/preprints/ESI-Preprints.html.

[14] E. M. Stein, Singular integrals and differentiability properties of functions, Princeton Math. Series, vol. 30, Princeton University Press, Princeton, NJ, 1970.

[15] Y. Meyer, Ondelettes et opérateurs, v. I, II, Hermann, Paris, 1990.

[16] J. Bourgain, "Bounded orthogonal systems and the $\Lambda(p)$-set problem", Acta Math., 162 (1989), 227-245.

[17] I. Daubechies, Ten Lectures on Wavelets, CBMS-NSF Regional Conference Series in Applied Math., vol. 61, SIAM, Philadelphia, PA, 1992.

[18] S. Campanato, "Proprieta di holderianita di alcune classi di funzioni", Ann. Scuola Norm. Sup. Pisa (3), 17 (1963), 175-188.

С.-Петербургское отделение Математического института им. В. А. Стеклова РАН

Поступило в редакцию e-mail: skis@pdmi.ras.ru 11 августа 2006 г.

Luleå University of Technology, Luleå, Sweden

e-mail: natan@sm.luth.se 